\title{
Mediastinoscopic tracheal resection and reconstruction under spontaneous-breathing anesthesia
}

\author{
Wenlong Shao, MD, PhD, Jianfei Shen, MD, Weiqiang Ying, MD, and Jianxing He, MD, PhD, FACS, \\ Guangzhou, China
}

\footnotetext{
From the Department of Thoracic Surgery, First Affiliated Hospital of Guangzhou Medical University; Guangzhou Research Institute of Respiratory Disease; and National Clinical Research Center for Respiratory Disease; and State Key Laboratory for Respiratory Disease, Guangzhou, China.

W.S. and J.S. contributed equally to this article.

Disclosures: Authors have nothing to disclose with regard to commercial support.

Received for publication Aug 27, 2015; revisions received Dec 22, 2015; accepted for publication Feb 2, 2016; available ahead of print March 5, 2016.

Address for reprints: Jianxing He, MD, PhD, FACS, Department of Thoracic Surgery, First Affiliated Hospital of Guangzhou Medical University, 151 Yan-jiang Rode, Guangzhou 510120, China (E-mail: drjianxing.he@ gmail.com).

J Thorac Cardiovasc Surg 2016;151:e105-7

$0022-5223 / \$ 36.00$

Copyright (c) 2016 by The American Association for Thoracic Surgery

http://dx.doi.org/10.1016/j.jtcvs.2016.02.013
}

The most common treatment for patients with upper tracheal tumors is a transcervical open approach under general intubated anesthesia. ${ }^{1}$ Mediastinoscopy is most commonly used in lymphadenectomy and lymph node biopsy in lung cancer. ${ }^{2}$ Here, we present a transcervical minimally invasive surgical method for treatment of a patient with tracheal adenoid cystic carcinoma (ACC) through mediastinoscopy under spontaneous-breathing anesthesia (SBA). We believe this treatment stimulated a quick patient recovery compared with traditional surgical methods. We obtained the patient's written informed consent for the procedure and subsequent use of data.

\section{CLINICAL SUMMARY}

An 18-year-old woman with a 6-month history of progressive exertion dyspnea and cough was referred to our hospital. She was also treated for asthma. Neck and chest computed tomography (CT) scan revealed a $1.2 \times 1.3 \times 1.4 \mathrm{~cm}$ spherical mass in the trachea, obstructing approximately $90 \%$ of the lumen (Figure $1, A$ and $B$ ). CT scan also revealed both subcutaneous and mediastinal emphysema. A mass in the trachea, $5 \mathrm{~cm}$ distal to the vocal cords, was found during bronchoscopic examination; biopsy was not performed because of surface bleeding of the mass.

The day after admission, the patient presented with sudden respiratory embarrassment. Emergency bronchoscopy revealed complete obstruction of the upper trachea. We proceeded by removing about $95 \%$ of the endotracheal tumor using a wire loop electrocautery for emergency relief of the patient's symptoms. There was $5 \mathrm{~cm}$ of tumor-free trachea distal to the remaining base of the tumor $(1 \mathrm{~cm}$ in diameter located on the right side) (Figure 1, C). Pathologic examination of resected pieces confirmed the diagnosis of ACC.

One month after the emergency procedure, pulmonary function tests were normal. Bronchoscopic examination was once again performed. Four locations of the bronchial

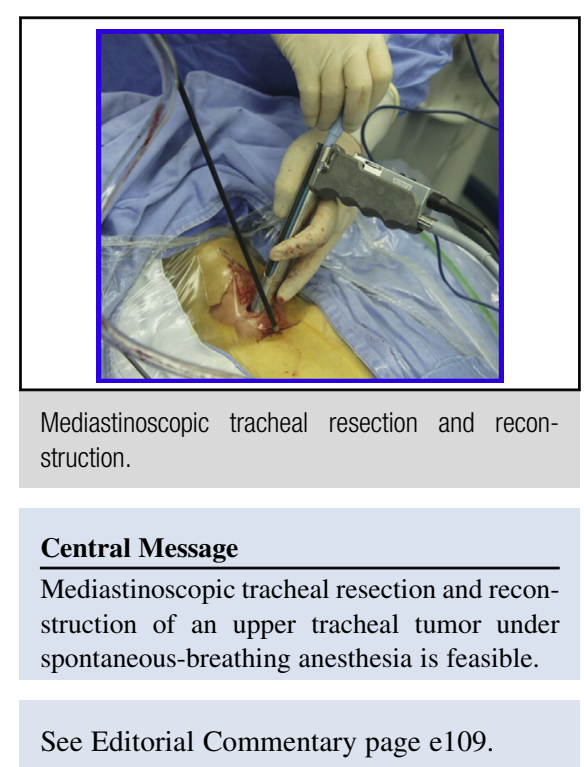

mucosa tissue were biopsied to identify the range of bronchial mucosa lesions (ie, carinal mucosa, $1.0 \mathrm{~cm}$ proximal to the tumor, $1 \mathrm{~cm}$ distal to the tumor, and left lateral wall opposite the tumor). No tumor was found. However, due to the incomplete resection of the tumor, surgery was proposed.

\section{TECHNIQUE}

The patient was placed in the supine position with cervical hyperextension. Nonintubated SBA without the use of muscle relaxants was performed. Details of the anesthesiologic procedure are described in previous reports. ${ }^{3}$ A laryngeal mask was used for oxygen support. A 3-cm incision was made at the suprasternal fossa along Langer's lines. Strap muscles were divided at the midline, the thyroid gland was retracted in the cranial direction, and a self-retracting wound protector (Lap-Protector, model FF0504, Hakko, Tokyo, Japan) was inserted into the cervical wound (Figure 2, A).

The video mediastinoscope was then introduced to assist in dissection of the surrounding tissue of the cervical and upper thoracic trachea (Figure 2, B).

A transtracheal needle was used under bronchoscopic view to determine the extent of trachea to be resected. The trachea was divided above and below the mass under mediastinoscopy. Four centimeters of the trachea was dissected followed by resection of $2 \mathrm{~cm}$. Meanwhile, 


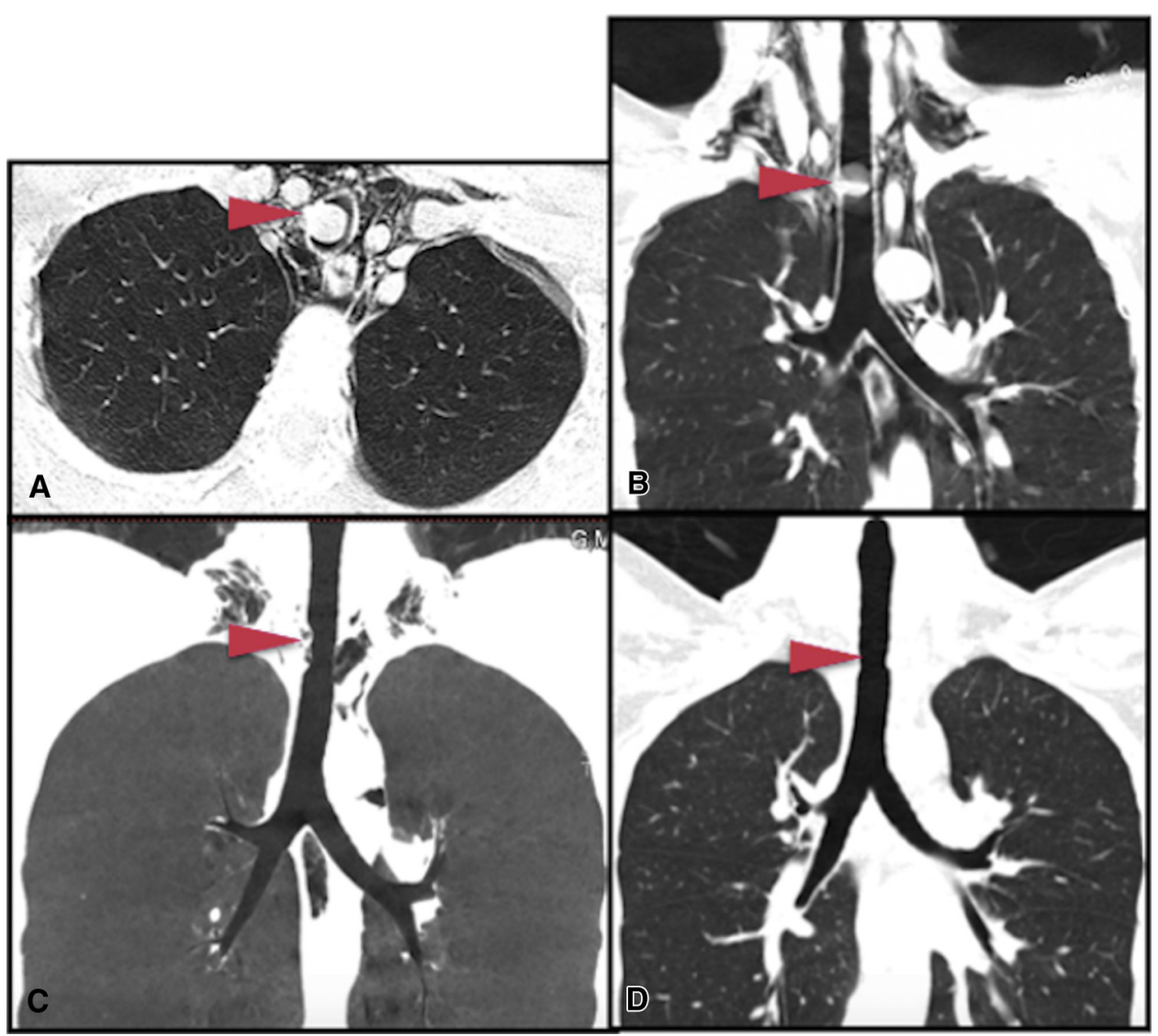

FIGURE 1. A and B, Neck and chest computed tomography scan revealed a $1.2 \times 1.3 \times 1.4 \mathrm{~cm}$ spherical mass in the trachea (arrow). C, The majority of the tumor was removed, but the base of the tumor remained. D, 2-month postoperative computed tomography scan.

a sterile nasal oxygen tube was placed at the lower part of the trachea, with an oxygen flow rate of $3 \mathrm{~L} / \mathrm{min}$ (Figure 2, C). Intraoperative frozen specimen examination revealed a result of negative surgical margins. Under media-stinoscopic vision end-to-end anastomosis was performed with 1 running suture, starting on the membranous part followed by the cartilaginous tissue, using 2-0 Prolene sutures (Ethicon Inc, Somerville, NJ). Salvage stitches were not needed. Intraoperative blood loss was $10 \mathrm{~mL}$ and operative time was 120 minutes.

Postoperative course was uneventful. Two hours after surgery, the patient walked back to the ward from the operating room and 3 hours later she resumed her normal diet. The patient was discharged on postoperative day 3 . The final pathologic examination confirmed the diagnosis of primary ACC of the trachea, with negative surgical margins.

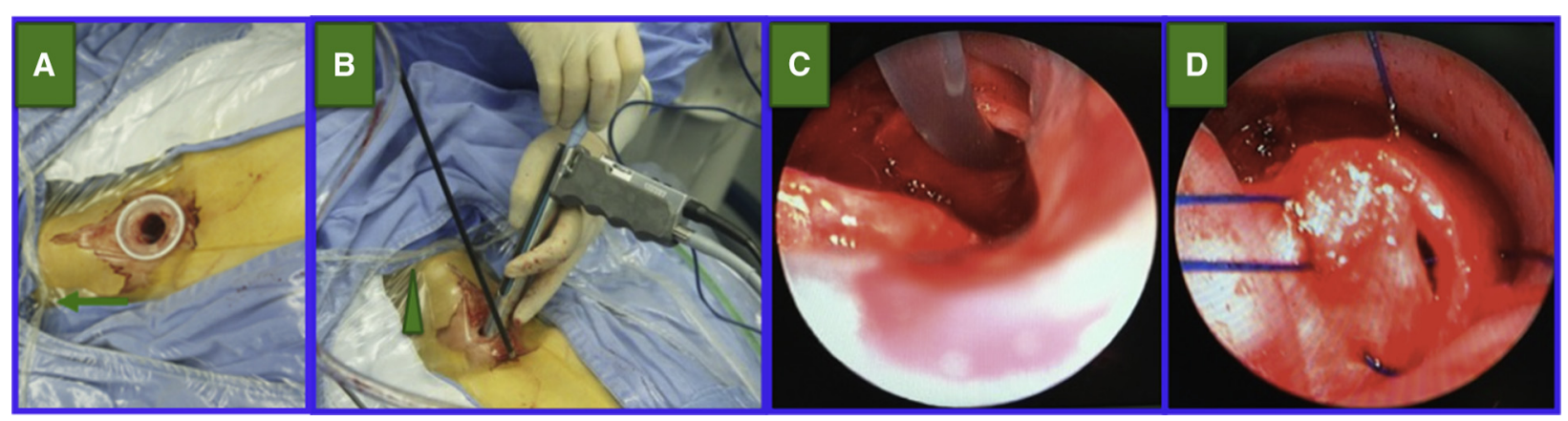

FIGURE 2. A, A self-retracting wound protector in the cervical wound. B, The mediastinoscope was then introduced. C, A sterile nasal oxygen tube was placed at the lower part of the trachea. D, Under mediastinoscopic vision, end-to-end anastomosis was performed with 1 running suture. Arrows point to head. 
At 2-month postoperative follow-up, recovery was uneventful. Bronchoscopic examination and CT scan confirmed no stenosis nor signs of recurrence (Figure 1,D).

\section{DISCUSSION}

Tracheal resection via mediastinoscopy is a novel technique. Primary ACC is usually managed by circumferential tracheal resection with primary anastomosis performed under an open surgical technique. ${ }^{1}$ In consideration of the young age and good physical condition of our patient, we decided to perform a mediastinoscopic tracheal resection. This minimally invasive approach provides the aesthetic advantage of minimal scarring. Moreover, the location and small size of the residual tumor $(<2 \mathrm{~cm}$ located in the upper trachea) made this procedure technically feasible.

Traditional anesthesia for tracheal procedures offers distinct challenges and requires careful coordination between surgical and anesthesia teams during airway excision and anastomosis. In our institution video-assisted thoracoscopic surgery under SBA has shown promising results. We have completed more than 1200 SBA video-assisted thoracoscopic surgery cases, including 200 anatomic lung resection cases wherein anatomical lung resections were performed. ${ }^{4}$ As a result of our previous experience we chose SBA for the tracheal tumor resection and reconstruction. This method benefits the patient by eliminating the negative effects generally associated with endotracheal intubation allowing the patient a faster recovery. ${ }^{5}$ We used a laryngeal mask to maintain blood oxygenation and ventilation and to decrease the risk of a need to transfer to tracheal intubation. During tracheal reconstruction, we used a sterile nasal oxygen tube in the field for additional oxygen support (Figure 2, C). Endotracheal ventilation and cross-field ventilation were still prepared as backup in case of an emergency situation such as hypoxemia during the surgery.
SBA may offer another advantage: The trachea might be more flexible, which may allow the surgeon more working space and mobility during resection. During similar procedures we have found that the addition of a wound protector creates more space for the operation. Resection and anastomosis were easily completed under mediastinoscopy. To facilitate the surgical procedure, we sometimes remove the outer structure of the video mediastinoscope leaving only the scope and light source, which provided more space for additional surgical instruments.

\section{CONCLUSIONS}

Mediastinoscopic tracheal resection and reconstruction under SBA is a feasible procedure. It may provide an alternative method for the treatment of upper tracheal tumors. We believe this method reduces surgical injury compared with the traditional open approach and as a result allows for faster recovery.

The authors thank Lindsey Hamblin for correcting the English spelling and grammar, as well as Keng Leong Ang and Lieven Depypere for providing additional comments on the content.

\section{References}

1. Behringer D, Könemann S, Hecker E. Treatment approaches to primary tracheal cancer. Thorac Surg Clin. 2014;24:73-6.

2. Turna A, Demirkaya A, Ozkul S, Oz B, Gurses A, Kaynak K. Video-assisted mediastinoscopic lymphadenectomy is associated with better survival than mediastinoscopy in patients with resected non-small cell lung cancer. J Thorac Cardiovasc Surg. 2013;146:774-80.

3. Guo Z, Shao W, Yin W, Chen H, Zhang X, Dong Q, et al. Analysis of feasibility and safety of complete video-assisted thoracoscopic resection of anatomic pulmonary segments under non-intubated anesthesia. J Thorac Dis. 2014;6:37-44.

4. Liu J, Cui F, He J. Non-intubated video-assisted thoracoscopic surgery anatomical resections: a new perspective for treatment of lung cancer. Ann Transl Med. 2015; $3: 102$.

5. Liu J, Cui F, Li S, Chen H, Shao W, Liang L, et al. Nonintubated video-assisted thoracoscopic surgery under epidural anesthesia compared with conventional anesthetic option: a randomized control study. Surg Innov. 2015;22:123-30. 